\title{
A Time Series Analysis of Structural Break Time in Ethiopian GDP, Export and Import
}

\author{
Hailegiorgis Biramo Allaro*
}

Department of Economics, Faculty of Business and Economics, Kotebe Metropolitan University, Addis Ababa, Ethiopia

\begin{abstract}
This research examined the structural break dates for GDP, export and import in Ethiopia using annual time series data spanning the years from 1974 through 2013. The study revealed that the economy of Ethiopia has been subject to a structural change and regime shift during the sample period. This paper reviews tests for structural change in linear regression models with Chow test which was formalized from Perron (1989) to perform tests on time series data on the sample period to determine the date(s) at which there was a statistically significant structural break. The study infers that endogenously determined structural break time for the GDP, export and import of Ethiopian economy was found to be 2003. Noteworthy, is that the structural break occurred after eleven years of the regime shift suggesting the policy change is not corresponding with the anticipated structural break in Ethiopian economy. This implies that structural break taking place endogenously well after policy announcement.
\end{abstract}

Keywords: Ethiopia; Export; GDP; Import; Structural break

\section{Introduction}

The methods of estimation of economic relationships and modeling fluctuations in economic activity have been subjected to fundamental changes in last four decades. Most of the work has concentrated on detecting the presence of structural break(s) and estimating the location of the break(s). However, there are two well-known problems with structural break estimation. The first one is the difficulty of differentiating data that is subject to a structural break (before and after which data shows stationary and trend stationary patterns) from data having a unit root. The second one is that although break locations in data can be estimated consistently, there is no efficiency condition for the limiting distribution of the estimates. Although consistency is a sufficient condition for the purpose of many empirical studies, efficiency could still be of interest if the aim is to obtain the smallest confidence intervals around the break dates. The stated reason behind these difficulties of estimating structural breaks is that the problem is nonstandard; a break date only appears under the alternative hypothesis, not under the null of no break. Perron empirical study makes a comprehensive review of both problems; however, it is very technical, and seemingly there is a lack of resources summarizing the relevant literatures [1]. To overcome these problems, Perron proposed allowing for a known or exogenous structural break in the Augmented Dickey-Fuller (ADF) tests [2].

The regime shift in Ethiopia took place in 1992 which intricately linked with the political macro-economy of Ethiopia. The following dates are considered important milestones:

\section{2: Policy change/political liberalization commenced}

\section{3: Devaluation of exchange rate}

\section{3: steady growth in Ethiopian economy.}

This sequence of political and economic events puts the dating problem into context. Should it be 1992-the date when political liberalization commenced, or 1993-the date of devaluation of exchange rate, or 2003 - the year when steady growth in Ethiopian economy was started?

The objective of this manuscript is to investigate empirical observation and related empirical research in addressing the problem of endogenously determined structural break time for GDP, export and import in Ethiopia. Conversely, as results of this study revealed, despite this research effort in identifying and examining the structural break time for the these variables in Ethiopia, there has been no study conducted on structural break time for GDP, exports and imports in the history of the country.

\section{Literature Review}

Structural break is an abrupt shift in a macroeconomic timeseries [3]. In recent years, economists and policy makers have been interested in understanding the nature of the stochastic properties of many macro-economic time series for structural break time of various economies. The main interest is to find out whether the macroeconomic time series is stationary or non-stationary in levels. The series that are non-stationary in levels, and hence their mean and variance are a function of time, for the effectiveness of economic policies, economic modelling and economic forecasting are enormous. A literature review in time-series econometrics reveals that there is a plethora of studies that examine the presence of a unit root in the macroeconomic time series of various countries [4-7].

There are different studies that examine the presence of structural break in the macroeconomic time series of various countries $[3,6]$. As there can be more than one break(s) in the data, the estimators can be further divided into two categories: (i) single break estimators and (ii) multiple break estimators. Actually, it is theoretically proven that consistency for the break date estimates is satisfied for single break estimators even if more than one break in the data exist $[8,9]$. This is done first finding one break in the data, and then splitting the data from there and searching for new breaks in the new samples.

${ }^{*}$ Corresponding author: Hailegiorgis Biramo Allaro, Department of Economics, Faculty of Business and Economics, Kotebe Metropolitan University, Addis Ababa, Ethiopia, Tel: +251911436809; E-mail: hailegiorgisbiramo@yahoo.com

Received May 06, 2018; Accepted August 01, 2018; Published August 09, 2018

Citation: Allaro HB (2018) A Time Series Analysis of Structural Break Time in Ethiopian GDP, Export and Import. J Glob Econ 6: 303. doi: 10.4172/2375 4389.1000303

Copyright: @ 2018 Allaro HB. This is an open-access article distributed under the terms of the Creative Commons Attribution License, which permits unrestricted use, distribution, and reproduction in any medium, provided the original author and source are credited. 
However, as there is no efficiency condition for any estimator, multiple break estimators are used to get more precise estimates, i.e., to find smaller confidence intervals around the breaks, and also to increase the rate of convergence to the break dates. This increases efficiency in the estimation of parameter values subject to the structural change. Conversely, Multi-Equations Systems are used to get more precise estimates for any type of estimator [10].

Quandt proposed likelihood ratio (LR) test statistics for an unknown change point, called Supremum (Max) test, while Andrews supplied analogous Wald and Lagrange Multiplier (LM) test statistics for it [11-13]. Then Andrews and Ploberger developed Exponential (LR, Wald and LM) and Average (LR, Wald and LM) tests [14]. These tests are calculated by using individual Chow Statistics for each date of the data except some trimmed portion from both ends of it. While the Supremum test is calculated for and finds the date that maximizes Chow Statistics, the most possible break point, the average and exponential tests use all the Chow statistic values and are only informative about the existence of the break but not its date. The deficiencies of the Supremum test are, however, as follows. It only has a power if one break occurs under the alternative hypothesis, and is valid as long as residuals from the regression follow (ibid). This means they do not show heterogeneity before and after the break, as is also a necessary condition for the Chow test. Heteroscedasticity and autocorrelation robust version of this test (also called Quandt Likelihood Ratio or Andrews-Quandt Statistics, which is the estimator used most commonly in this literature) can be used, even though it still gives the most possible break date (it is so because of small sample properties). It also strongly suffers from large confidence intervals around the break date [14].

The single break model test was applied by Bai and Perron for quasi likelihood estimation in a vector auto regression (VAR) setting [10]. They showed that with common breaks across equations, the precision of the estimates' increases with the number of equations in the system. However, their methodology obviously can only be carried out as long as equations are expected to show a break in the same time period. This could be the case when several variables are co-integrated. Besides, this test is designed for a single break and there could be more than one break date in the data, in which case these test exhibits non-monotonic power function $[15,16]$ following the work of Bai and Perron, first define minimum segment length (in proportion to the total data) [17]. Given this constraint, they then search for the optimal partition of all likely segments of data to obtain global minimizers of the sum of squared residuals. By this way, they obtain the location of breaks, minimizing their objective function for any possible number of breaks. Then they sequentially test for whether an additional break date significantly reduces the sum of squared errors. Their methodology inherits both pure and partial structural change models, which consistently identify the break dates Perron [1]. This is because when estimating a single break model in the presence of multiple breaks the estimate of the break fraction will converge to one of the true break fractions. The one which is dominant in the sense that taking it into account allows the greatest reduction in the sum of squared residuals (in the case of two breaks that are equally dominant), then the estimate will converge with probability half $(1 / 2)$ to either break. The comment on this procedure states the fact so as to the method of estimation is based on the leastsquares principle, which implies that even if changes in the variance of error terms are allowed, provided they occur at the same dates as the breaks in the parameters of the regression, such changes are not exploited to increase the precision of the break date estimators. This is because the fact that the least-squares method imposes equal weights on all residuals allowing different weights, as needed when accounting for changes in variance, which requires adopting a quasi-likelihood framework.

Perron and Qu bring a novel approach to structural break analyses, which enable them to find considerably small confidence intervals around the break dates [18]. Perron and Qu use a multiple equation model. They first define the minimum segment length of the data that could be separated with breaks [18]. Given this constraint, they then search for the optimal partition of all possible segments of data which the model fit, where the objective function being maximized is a quasilikelihood one based on normal errors.

Hendry and Massmann applied to test unit roots under structural breaks, or directly to test for structural breaks, which rests on the principle that there is an appropriate combination of variables, having a break in common, that does not display the breaks any longer [3]. Moreover, this very reason also prevents co-feature analysis from always being applicable. Applying them requires using more than one series, which are suspected to have common breaks. In order to deal with breaks in the growth rate of export, import and GDP, it requires using different types of regressions and cannot be tested at a time with co-breaking analysis. Alternatively, each variable may have been tested for a break independently from the other [1].

Thus, most of the studies have concentrated on detecting the presence of a structural break(s) and estimating the location of the break(s) as well as the values of the model parameters prior to and following break(s) [1]. However, there are two well-known problems with structural break estimation. The first is the difficulty of differentiating data that is subject to a structural break. The second is that although break locations in data can be estimated consistently, there is no efficiency condition for the limiting distribution of the estimates. Although consistency is a sufficient condition for the purpose of many empirical studies, efficiency could still be of interest if the aim is to obtain the smallest confidence intervals around the break dates. The stated reason behind these difficulties of estimating structural breaks is that the problem is nonstandard; a break date only appears under the alternative hypothesis, not under the null of no break [1].

The empirical study on the structural break indicates that it occurs in many time series analysis for a number of reasons, including economic crises, changes in institutional arrangements, policy changes and regime shifts. The study carried out by Perron, 1989; 1997; Leybourne and Newbold, 2000 specified that, if structural changes are present in the data generating process but not incorporated in the unit root test specification, results may be biased towards flawed non-rejection of the non- stationarity hypothesis $[9,19]$. The outcome of such a result in turn implies that any shock - whether demand, supply, or policyinduced - to the variable will have effects on the variable into the very long run. This also may affect accuracy in forecasting.

Many empirical studies were dealt with examining the order of integration of output series in advanced economies and the Organization for Economic Co-operation and Development (OECD) countries. Some of these famous studies include the empirical investigations conducted by the authors $[4,5]$. These studies found that the macroeconomic time series of many advanced countries, including many OECD countries to be non-stationary. Rapach (2002) concluded that the series are integrated of order one, I (1). In contrast, empirical investigations by Gaffeo and Gallagati (2005) concluded that the series are stationary, and hence they are integrated of the order zero, I (0) [5]

Even though, in the literature there exist many empirical investigations of structural break time for the time-series properties 
of the macroeconomic time series for developed countries, one finds that there are limited number of studies that examine the phenomenon under consideration for developing countries, especially African economies. The only study on the time-series properties of the African economies is found by Chang et al. (2005). They used the data of 26 select African countries for the period 1960-2000 and concluded that for the majority of the countries the series are non-stationary. Another study undertaken by Romero-Avila tests the unit root hypothesis for a panel of 46 African countries over the period 1950-2001 using the data from Penn World Table (2006). He concluded that the macroeconomic series in these African countries experienced multiple breaks and a regime wise trend stationary.

However, these endogenous tests were criticized for their treatment of breaks under the null hypothesis. They do not allow for a break(s) under the null hypothesis of unit root and derive their critical values accordingly. So they exclude the possibility that there may be a unit root process with a break. One way of overcoming this problem would be taking to log difference of the data, which made the series stationary, and look for a break in the growth rate of the series. Nonetheless, it would be wise to avoid data conversions that smooth the data especially when the data is not long enough or includes outliers. It is because under these conditions break estimation tends to catch any kind of one- time deviation in the data rather than finding a change in trend or in a mean. In this case, these tests declare data as stationary with breaks.

Structural break tests can be divided into three categories: (i) the Chow Test, used to test whether the series has a break in the tested date; (ii) look for the presence of a break in the series, which may exist at any time within the sample period. Some tests in this category also reveal the most possible break date as a by-product, and (iii) estimators, which first estimate the unknown date of the break, then test it. For any type of break, the date of the break, if it exists, is unknown so that it falls into the third category [20]. However, to understand the basics of the structural break estimators that are used to find unidentified break dates and test them, it is better to start with the Chow Test. It is because mysterious date estimators that use more complicated tests basically rest on the same principles as this test. Chow Tests look for the following: whether splitting data from the possible break point and estimating two generated sub-samples separately by least square gives significantly better than using the whole sample at once; if the answer is yes, the null hypothesis of no break is rejected. The resulting statistics would be F-statistics, log likelihood ratio or the Wald statistic [3].

Though, as there can be more than one break in the data, the estimators can be further divided into two categories: (i) single break estimators and (ii) multiple break estimators. Actually, it is theoretically proven that consistency for the break date estimates is satisfied for single break estimators even more than one break in the data exist. These works by first finding one break in the data, and then splitting the data from there and searching for new breaks in the new samples. However, as there is no efficiency condition for any estimator, multiple break estimators are used to get more precise estimates, i.e., to find smaller confidence intervals around the breaks, and also to increase the rate of convergence to the break dates. This increases efficiency in the estimation of parameter values subject to the structural change. Conversely, Multi-Equations Systems are used to get more precise estimates for any type of estimator [10]

For the unknown break date, Quandt proposed likelihood ratio test statistics for an unknown change point, called Supremum (Max) Test, while Andrews supplied analogous Wald and Lagrange Multiplier test statistics for it [11-13]. Then Andrews and Ploberger developed
Exponential (LR, Wald and LM) and Average (LR, Wald and LM) tests [14]. These tests are calculated by using individual Chow Statistics for each date of the data except some trimmed portion from both ends of it. While the Supremum test is calculated for and finds the date that maximizes Chow Statistics, the most possible break point, the Average and Exponential tests use all the Chow Statistic values and are only informative about the existence of the break but not its date. The deficiencies of the Supremum test are, however, as follows. It only has a power if one break occurs under the alternative hypothesis, and is valid as long as residuals from the regression follow ibid. This means they do not show heterogeneity before and after the break, as is also a necessary condition for the Chow Test. Heteroscedasticity and autocorrelation robust version of this test (also called Quandt Likelihood Ratio or Andrews-Quandt Statistics, which is the estimator used most commonly in this literature) can be used, even though it still gives the most possible break date (it is so because of small sample properties). It also strongly suffers from large confidence intervals around the break date $[13,14]$.

The single break model test was applied Bai and Perron for quasi likelihood estimation in a VAR (vector auto regression) setting [10] They showed that with common breaks across equations, the precision of the estimates' increases with the number of equations in the system. However, their methodology obviously can only be carried out as long as equations are expected to show a break in the same time period. This could be the case when several variables are co-integrated. Besides, this test is designed for a single break and there could be more than one break date in the data, in which case these test exhibits non-monotonic power function $[15,16]$.

Perron and $\mathrm{Qu}$, following the work of Bai and Perron, first define minimum segment length (in proportion to the total data) $[17,21,22]$. Given this constraint, they then search for the optimal partition of all likely segments of data to obtain global minimizers of the sum of squared residuals. By this way, they obtain the location of breaks, minimizing their objective function for any possible number of breaks. Then they sequentially test for whether an additional break date significantly reduces the sum of squared errors. Their methodology inherits both pure and partial structural change models, which consistently identify the break dates Perron [1]. This is because when estimating a single break model in the presence of multiple breaks the estimate of the break fraction will converge to one of the true break fractions.

The one which is dominant in the sense that taking it into account allows the greatest reduction in the sum of squared residuals (in the case of two breaks that are equally dominant), then the estimate will converge with probability half $(1 / 2)$ to either break. The comment on this procedure states the fact so as to the method of estimation is based on the least-squares principle, which implies that even if changes in the variance of error terms are allowed, provided they occur at the same dates as the breaks in the parameters of the regression, such changes are not exploited to increase the precision of the break date estimators. This is because the fact that the least-squares method imposes equal weights on all residuals allowing different weights, as needed when accounting for changes in variance, which requires adopting a quasilikelihood framework.

Finally, bring a novel approach to structural change analyses, which enable them to find considerably small confidence intervals around the break dates. Use a multiple equation model [18]. They first define the minimum segment length of the data that could be separated with breaks. Given this constraint, they then search for the optimal partition of all possible segments of data which the model fit, where the objective 
function being maximized is a quasi-likelihood one based on normal errors.

\section{Model Specification}

A series of data can often contain a structural break, due to a change in policy or sudden shock to the economy. The $\mathrm{F}$ test and Chow test were applied to test the existence of endogenously determined structural break time on three macroeconomic variables in the study period. Thus, the study signifies structural break with adopted Chow test of Perron structural break analysis model [9]. In this case each model specifies just a single regression line to fit the data points (scatter plot), which can be expressed as:

$$
\begin{aligned}
& \mathrm{GDPs}_{\mathrm{t}}=\alpha_{0}+\alpha_{1} \operatorname{logGDP} \mathrm{P}_{1}+\alpha_{2} \operatorname{logGDP} \mathrm{P}_{2}+\ldots \alpha_{\mathrm{t}} \operatorname{logGDP} \mathrm{P}_{\mathrm{t}}+\mu_{\mathrm{t}} \\
& \mathrm{XS}_{\mathrm{t}}=\alpha_{0}+\alpha_{1} \log \mathrm{x}_{1}+\alpha_{2} \log \mathrm{x}_{2}+\ldots+\alpha_{\mathrm{t}} \log \mathrm{x}_{\mathrm{t}}+\mu_{\mathrm{t}} \\
& \mathrm{ms}_{\mathrm{t}}=\alpha_{0}+\alpha_{1} \log \mathrm{m}_{1}+\alpha_{2} \log \mathrm{m}_{2}+. .+\alpha_{\mathrm{t}} \log \mathrm{m}_{2}+\mu_{\mathrm{t}}
\end{aligned}
$$

Where, GDPs $x s_{t} m s_{t}$ refers structural break time to GDP; export; and import; respectively; GDP, gross domestic product; $x$, value of exports; and $\mathrm{m}$, value of imports in million USD; a's are unknown parameters to be estimated, $\mathrm{t}$, is time in years (1974-2013 and $\mu$ is random terms that are independently and identically distributed with mean zero and variance ${ }^{2}\left(\delta^{2}\right)$.

Regressions were run for all the variables in the sample period. Then tests for a structural break were done that involves testing whether the coefficients on $\alpha_{t} \log G D P ; \alpha_{t} \log x_{t}$ and $\alpha_{t} \log m_{t}$ were significantly different from zero. To test the hypothesis $H_{0}: \alpha=$ structural stability versus $H_{1}: \alpha=$ structural break, regression of RSS (regression using all the data, before and after the structural break), RSS (regressions on the data before the structural break and RSS (regressions on the data after the structural break) was done.

\section{Results and Discussions}

\section{Graphic analysis}

The graphical representation of real GDP, export and import data pre reform indicates that there is only cyclical variation without structural break time in the macroeconomic variables (Figure 1).

The graphical representation of real GDP, export and import data for the post reform period signifies that the actual structural break date for the variables was found to be 2003. This implies that, although, endogenously determined regime shift date was 1992; it took 11 years to bring structural break in Ethiopian macroeconomic variables [23]. This is presented in the following Figure 2.

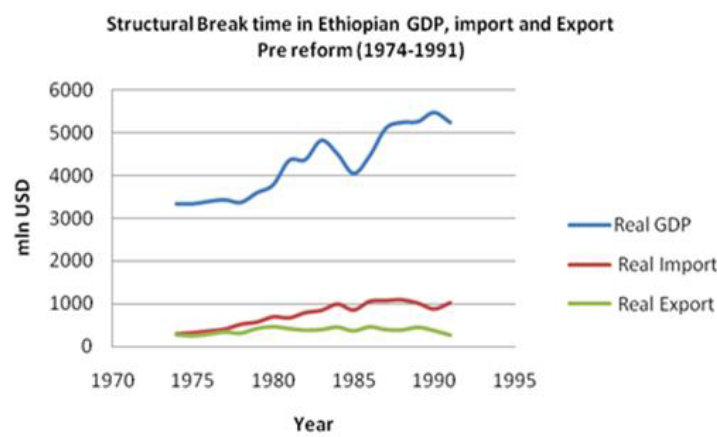

Figure 1: Structural break time in the Ethiopian macroeconomic variables, pre reform (1974-1991).

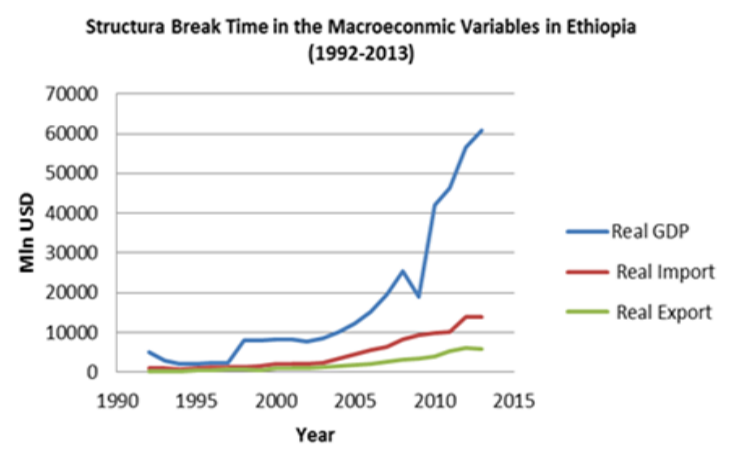

Figure 2: Structural break time in the Ethiopian macroeconomic variables, pre reform (192-2013)

\section{Econometric analysis}

The econometric analysis was applied to investigate the structural break time on three macroeconomic variables. The econometric analysis begins with the testing of variables for unit roots to determine whether they can be considered as a stationary or non-stationary process [24]. The tests showed that the variables were stationary at the second difference. Critical values for tests were found to be -3.55 ; and 3.21 at $5 \%$ and $10 \%$ respectively. Then, it was analyzed with the F-test for a restriction (Tables 1-3).

Based on these outputs the test statistic was calculated using the following formulae:

$$
F=\frac{R S S-\left(R S S_{1}+R S S_{2}\right) / K}{\left(R S S_{1}+R S S_{2}\right) /\left(N_{1}+N_{2}-2 K\right)}
$$

The critical value for $\mathrm{F}(6,30)$ is 2.42 at $5 \%$ significance level. This implies that the test statistic (9.68) is greater than the $95 \%$ critical value (2.42) of F-test; it is possible to reject the null of no structural break times in macroeconomic variables under investigation. The analysis with F-test estimation technique indicates that there is structural break time for the variables under investigation. The Chow Test results on the regressions on the order of the I (1) variables, GDP, export and import indicates that the only significant breakpoint was found to be 2003 with p-value or significance $=0.00$ or better. Thus, there was a structural break in the series in 2003 (Table 4).

The issue of structural break is of considerable importance in the analysis of macroeconomic time series. It occurs in many time series for any number of reasons, including economic crises, changes in institutional arrangements, policy changes and regime shifts [1]. The Ethiopian economy has been subjected to a structural change and twofold regime shifts during the sample period. The order of integration for GDP, exports and imports were examined with the application of conventional unit root tests of ADF (Augmented Dickey- Fuller) time series properties of data [2]. The F-statistics and Chow Test were used to determine endogenously the more likely time of structural break(s) in macroeconomic variables of the Ethiopian economy. Based on the models, the presence of unknown structural break(s) time in the data was considered. After running the F-test for the presence of structural break; the results from the F-test clearly indicate that for the series under examination; the null hypothesis of no structural break time was rejected. In other words, the empirical results based on the model indicated that there was structure break which provided adequate evidence to reject the null hypothesis of no structural break for any of the variables under investigation. The empirical results designated 


\begin{tabular}{|c|c|c|c|c|}
\hline Mode & Sum of Squares & df & Mean Square & F \\
\hline Regression & 3773.330 & 3 & 1257.777 & 360.427 \\
\hline
\end{tabular}

Table 1: RSS (residual sum of squares) for all data.

\begin{tabular}{|c|c|c|c|c|}
\hline Mode & Sum of Squares & df & Mean Square & Sig. \\
\hline Regression & 1973.165 & 3 & 657.722 & 289.311 \\
\hline Residual & 56.835 & 25 & 2.273 & \\
\hline Total & 2030.000 & 28 & & \\
\hline RSS $1=56.835$ & & & & \\
\hline
\end{tabular}

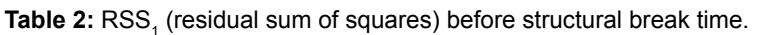

\begin{tabular}{|c|c|c|c|c|}
\hline Mode & Sum of Squares & df & Mean Square & F \\
\hline Regression & 27.983 & 3 & 9.328 & 1603.446 \\
\hline Residual & 0.017 & 3 & 0.006 & \\
\hline Total & 28.000 & 6 & & \\
\hline RSS $=0.017$ & & & \\
\hline
\end{tabular}

Table 3: $\mathrm{RSS}_{2}$ (residual sum of squares) after structural break time.

\begin{tabular}{|c|c|c|c|c|c|c|}
\hline Description & Sample period & Variable & Significant year & \multicolumn{3}{|c|}{ Tests and probability } \\
\cline { 3 - 6 } & & & & F-statistic & probability & Log likelihood ratio \\
\hline GDP & $1974-2013$ & probability & 0.000 & -5.783486 \\
\hline Export & $1974-2013$ & logGDP & 2003 & 28.02335 & 0.000 \\
\hline Import & $1974-2013$ & Logmt & 2003 & 26.01631 & 0.000 & -4.564348 \\
\hline
\end{tabular}

Table 4: Chow test on regression of export and import (1974-2013).

that for the variables under investigation the endogenously determined break date was 2003, which closely corresponds to the important phenomena in the performance of the Ethiopian economy. Therefore, these important phenomena in Ethiopian economy lead to analyze the historical events of the macroeconomic sphere of the country.

\section{Conclusion}

This research examined the structural break dates for GDP, exports and imports in Ethiopia. The conventional unit root tests of ADF (Augmented Dickey-Fuller) time series properties of data were analyzed to determine the order of integration [2]. Based on the F-statistics, the presence of unknown structural break(s) time in the data was considered. After accounting for the presence of structural break the results from the F-test clearly indicate that for the series under examination, the null hypothesis of no structural break time was rejected. The empirical results designated that for the variables under investigation the endogenously determined break date was 2003, which closely corresponded to the important phenomena in the performance of the Ethiopian economy. Therefore, the sudden change in Ethiopia macroeconomic variables was happened in 2003 because of the combination of factors, including good weather, increased efforts in agricultural extension, increased usage of fertilize, and foreign capital inflows which funded major increases in private and public infrastructure investments, political stability and SAP (structural adjustment programme) that liberalized foreign trade and realized other reforms in monetary and fiscal spheres.

\section{References}

1. Perron $P$ (2005) Dealing with Structural Breaks. Econometric Theory.

2. Dickey DA, Fuller WA (1979) Distributions of the Estimators for Autoregressive Time Series with a Unit Root. J Am Stat Assoc 74: 427-481.

3. Hendry DF Massmann M (2007) Co-Breaking: Recent Advances and a Synopsis of the Literature. J Bus Econ Stat, Am Stat Assoc, 25: 33-51.

4. Gerdtham UG, Lothgren M (2000) On Stationarity and Cointegration of International Health Expenditure and GDP. J Health Econ 19: 461-475.
5. Gaffeo EM, Gallagati (2005) Requiem for the Unit Root in Per Capita Real GDP? Additional Evidence from Historical Data . Empirical Economics 30: 37-63.

6. Glynn J, Perera N, Verma R (2007) Unit Root Tests and Structural Breaks: A Survey with Applications. J Quant Methods Econ Bus Adm 3:63-79.

7. Silverstre $\mathrm{Cl}$, Lluis $\mathrm{J}(2005)$ Health care expenditure and GDP: are they broken stationary? Journal of Health Economics, 24: 939-854.

8. Bai J (1997) Estimating multiple breaks one at a time. Econometric Theory. New York, USA, 13: 315-352.

9. Perron $P$ (1989) The great crash, the oil price shock, and the unit root hypothesis. Econometrica 57: 1361-1401.

10. Bai J, Perron P (1998) Estimating and Testing Linear Models with Multiple Structural Changes. Econometrica 66: 47-78.

11. Quandt RE (1958) The estimation of the parameters of a linear regression system obeying two separate regimes. Journal of the American Statistical Association 53: 873-880.

12. Quandt RE (1960) Tests of the hypothesis that a linear regression system obeys two separate regimes. J Am Stat Assoc 55: 324-330.

13. Andrews DWK (1993) Tests for parameter instability and structural change with unknown change point. Econometrica, USA 71: 395-397.

14. Andrews DWK, Ploberger W (1994) Optimal Tests when a Nuisance Parameter is Present Only under the Alternative. Econometrica USA 62: 1383-1414.

15. Vogelsang TJ (1997) Wald-type tests for detecting breaks in the trend function of a dynamic time series. Econometric Theory 13: 818-849.

16. Vogelsang TJ (1999) Sources of nonmonotonic power when testing for a shift in mean of a dynamic time series. J Econom 88: 283-299.

17. Perron P, Qu Z (2006) Estimating Restricted Structural Change Models. J Econom 134: 373-399.

18. Perron P, Qu Z (2007) Estimating and Testing Structural Changes in Multivariate Regressions. Econometrica, J Econom Soc 75: 459-502.

19. Perron P (1997) Further Evidence on Breaking Trend Functions in Macroeconomic Variables, J Econom, 80: 355-385.

20. Chow GC (1960) Tests of Equality between Sets of Coefficients in Two Linear Regressions. Econometrica 28: 591-605.

21. Bai J, Perron $P$ (2003) Computation and analysis of multiple structural change models. J Appl Econ New York, USA, 18: 1-22. 
Citation: Allaro HB (2018) A Time Series Analysis of Structural Break Time in Ethiopian GDP, Export and Import. J Glob Econ 6: 303. doi: 10.4172/23754389.1000303

Page 6 of 6

22. Bai J, Perron P, (2003). Critical values for multiple structural change tests. Econom J New York, USA, 6: 72-78.
23. Christiano LJ (1992) Searching for breaks in GNP. J Bus Econ Stat 10: 237-250. 24. NBE (National Bank of Ethiopia), Annual Reports of various years'. 